\title{
A Haptic Enabled Multimodal Pre-Operative Planner for Hip Arthroplasty
}

\author{
S. Imboden ${ }^{*}$, M. Petrone ${ }^{*}$, P. Quadrani ${ }^{*}$, C. Zannoni ${ }^{*}$, R. Mayoral ${ }^{* \dagger}$, G. J. Clapworthy ${ }^{* \dagger,}$ D. \\ Testi $^{\ddagger}$, M. Viceconti ${ }^{\ddagger}$, D. Neiberg ${ }^{ \pm}$, N.G.Tsagarakis ${ }^{* \star}$ and D.G.Caldwell ${ }^{* \star}$ \\ (*)CINECA, Italy \\ (*i)Department of Computing \& Information Sciences, University of Luton, \\ (†)Laboratorio di Tecnologia Medica, Istituti Ortopedici Rizzoli, Bologna, Italy \\ ( \pm Department of Speech, Music and Hearing, KTH, Sweden \\ $(*)$ School of Computer Science and Engineering, University of Salford, UK \\ E-mail:,m.petrone@cineca.it, p.quadrani@cineca.it,c.zannoni@cineca.it, \\ n.tsagarakis@salford.ac.uk,neiberg@speech.kth.se
}

\begin{abstract}
This paper introduces the Multisense idea, with a special reference to the use of Haptics in the medical field and, in particular, in the planning of total hip replacement surgery. We emphasise the integration of different modalities and the capability of the multimodal system to gather and register data coming from different sources.
\end{abstract}

\section{Introduction}

Improvements in computer science and reductions in computational costs have, in recent years, allowed the consideration of specialized Virtual Reality (VR) approaches to medical applications. In particular, the use of multimedia environments have become of great interest in the training of clinical personnel. VR techniques have been already used in neuro, craniofacial , and orthopaedic surgery.

Pre-operative planning is a fundamental phase in total hip replacement (THR) surgery [1] and the advantage of using a 3D environment has been demonstrated [2]. Different systems for preoperative planning of THR have been developed in the last few years however, although it is more intuitive to grasp and examine a virtual object within an immersive system, the navigation is mostly achieved by using a mouse (with two degrees of freedom) and a flat (2D) screen for pseudo-3D interaction. That strategy is questionable, since it has been proved that accuracy of positioning the implant using 3D preoperative planning software is strongly effected by the graphical user interface[3].
In addition, there has been limited exploitation of other modalities such as haptics and speech, and the benefits that can be achieved by such integration are still largely unexplored. From this rationale, the Multisense project started with the aim of combining different sensorial devices (haptics, speech, visualization, tracking) in a unique VR environment for pre-operative planning of total hip replacement. While the demonstrator of the multisensorial integration is focused on a specific clinical application, the effectiveness of this approach, after validation, will be of great relevance also for many other orthopedic and medical applications.

\section{System Implementation}

The hardware implementation of the multimodal system for the clinical application of the pre-operative planning of total hip replacement is presented in Fig.1. To address the intensive computation needs of a such a multimodal system and particularly to accommodate the different updates rates required from the control of the different cues (e.g speech subsystem produces inputs at rates of a few seconds, graphics systems at the rate of $10-30 \mathrm{msec}$ while haptic devices at the speed of fraction of millisecond) the multimodal architecture uses multiple networked dedicated machines. Advantage of this approach over a single machine multi-threaded approach include:

i) Minimum coupling between local rates running on different machines providing

ii) More consistent rates for critical process such as the haptic servo input/feedback control process and as a result this enables 
iii) Stable haptic rendering of more complex environments

iv) Provision of functionally separate, extensible and reconfigurable control of the different input feedback subsystems.

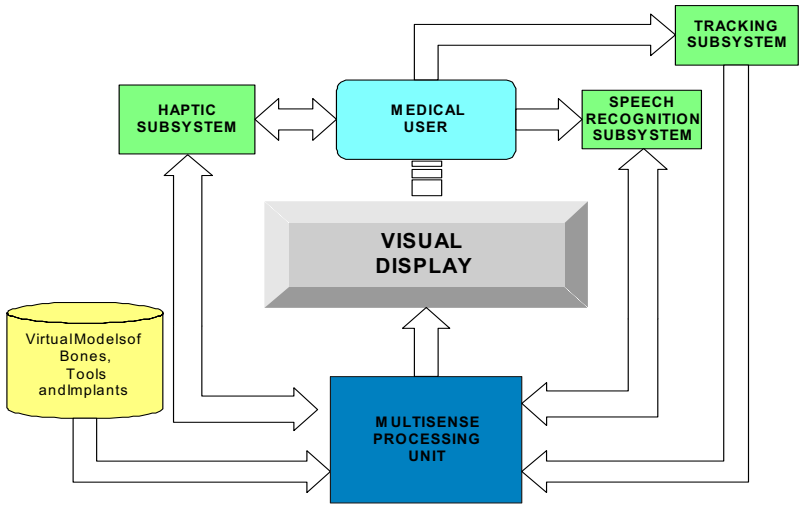

Fig.1. Multimodal system implementation.

The price for the above is of course paid by the increased synchronization necessities between the various subsystems implemented on the different machines. Base on this multiple networked machines approach a dedicated graphics server machine is used to perform the hip replacement application graphics rendering. The low servo control and the input/feedback coordination of the haptic subsystem are managed by the haptic server machine while a third server machine is responsible for the control of the speech subsystem. The communication between the various machines is performed using a $\mathrm{TCP} / \mathrm{IP}$ protocol interface.

\section{The MAF application framework}

The Multimod Application Framework (MAF) used in this planner application is a software library allowing the rapid development of computer aided medicine applications, more recently also used to develop visualization applications in other fields of scientific visualization. In its most general incarnation the MAF supports the so-called Multimodal Display and Interaction (MDI) paradigm, in which the man-machine interface is multimodal and multisensorial.

A generic MAF application is composed by components, which control system resources, that can be subdivided into data entities and application services. We call a data entity the Virtual Medical Entity or VME for short. The application services can be distinguished in Views, Operations, GUIs and Devices. Formally, a component is an instance of a particular $\mathrm{C}++$ class. Every MAF application is an instance of the Logic component. The main role of Logic is to control the communication between the underlying components to make them follow a consistent application's logic.

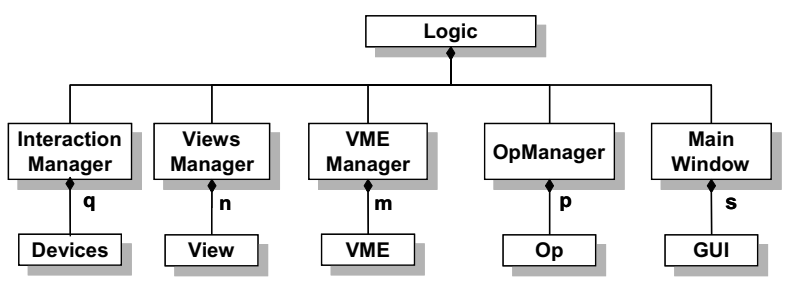

Fig.2. MAF architecture diagram.

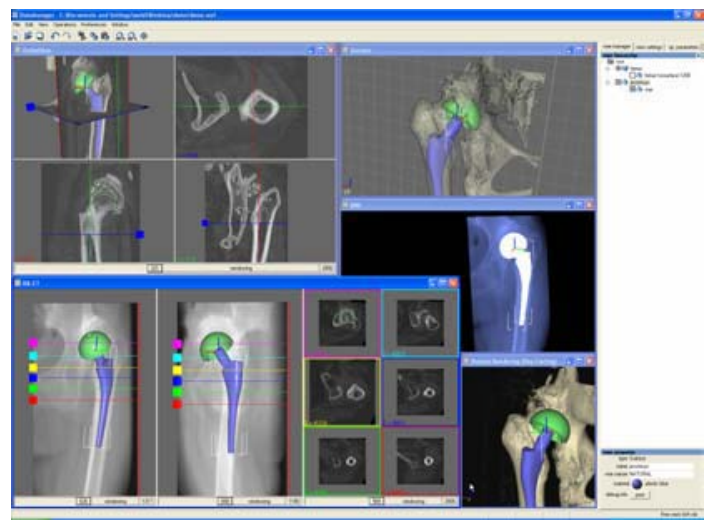

Fig.3. MAF Multi display paradigm.

MAF is able to manage interaction with multiple $\mathrm{I} / \mathrm{O}$ devices, and to perform this it implements routing, locking, and fusion mechanisms through a component named Interaction Manage. For Haptic devices, requiring very high and decoupled update rates, high speed loops are running inside the Haptic subsystem

\section{ACKNOWLEDGEMENTS}

This work has been supported through the EU under contract IST-2001-34121.

\section{References}

[1] C.G. Schizas, B. Parker, and P.-F. Leyvraz, "A study of preoperative planning in CLS total hip arthroplasty", Hip International 1996;6:75-81.

[2] M. Viceconti, R. Lattanzi, B. Antonietti, S. Paderni, R. Olmi, A. Sudanese, and A. Toni, "CT-based surgical planning software improves the accuracy of THR preoperative planning", Medical Engineering \& Physics 2003;25(5):371-377.C.G. Schizas, B. Parker, and P.-F. Leyvraz, "A study of pre-operative planning in CLS total hip arthroplasty", Hip International 1996;6:75-81.

[3] M. Viceconti, R. Lattanzi, C. Zannoni, and A. Cappello, "Effect of the display modality on the spatial accuracy of orthopaedic surgery pre-operative planning applications", Medical Informatics and the Internet in Medicine 2002;27(1):21-32. 